\title{
Germanica
}

14 | 1994

Les Fictions d'actualité dans les pays de langue allemande au XXe siècle

\section{Wider den kitschigen Sozialismus Hermann Brochs Kritik an der Tendenzkunst und seine «polyhistorische» Antwort}

La critique de la littérature tendancieuse formulée par Hermann Broch et sa réponse "polyhistorique»

Till R. Kuhnle

\section{(2) OpenEdition}

\section{Journals}

Édition électronique

URL : http://journals.openedition.org/germanica/2210

DOI : 10.4000/germanica.2210

ISSN : 2107-0784

Éditeur

Université de Lille

\section{Édition imprimée}

Date de publication : 1 décembre 1994

Pagination : $61-78$

ISSN : 0984-2632

\section{Référence électronique}

Till R. Kuhnle, «Wider den kitschigen Sozialismus Hermann Brochs Kritik an der Tendenzkunst und seine «polyhistorische» Antwort », Germanica [Online], 14 | 1994, Online erschienen am: 20 Januar 2014, abgerufen am 06 Oktober 2020. URL : http://journals.openedition.org/germanica/2210 ; DOI https://doi.org/10.4000/germanica.2210

Ce document a été généré automatiquement le 6 octobre 2020.

(c) Tous droits réservés 


\section{Wider den kitschigen Sozialismus Hermann Brochs Kritik an der Tendenzkunst und seine «polyhistorische» Antwort}

La critique de la littérature tendancieuse formulée par Hermann Broch et sa réponse "polyhistorique»

Till R. Kuhnle

1 Als Philosoph gehörte der Schriftsteller Hermann Broch ${ }^{1}$ nicht zu denjenigen Autoren, die theoretische Abstinenz üben. Ausführlich thematisierte er das Verhältnis von Kunst und Literatur zu ihrer Zeit. Seine Romantrilogie Die Schlafwandler ${ }^{2}$ steht im engen Kontext zur Vorgeschichte der Epoche ihrer Entstehung, zur deutschen Geschichte bis 1918. Mit dem letzten Roman dieser Trilogie, 1918 Hugenau oder die Sachlichkeit, reihte sich Broch unter die großen Autoren des experimentellen Romans ein. Sein poetologischer Anspruch ist der Roman als Residuum von Totalitätserfahrung unter den epistemlogischen Prämissen seiner Epoche, die ein von Lukâcs aufgeworfenes Problem des Romans noch stärker hervortreten lassen und an die Grenze der Gattung zu führen scheinen:

Die Dissonanz der Romanform, das Nicht-eingehen-Wollen der Sinnesimmanenz in das Leben, gibt ein Formproblem auf, dessen formeller Charakter viel verdeckter ist, als der anderer Kunstformen, das wegen seiner Inhaltlichkeit vielleicht noch ausgesprocheneres Zusammenwirken von ethischen und ästhetischen Kräften erfordert, als es bei evident reinen Formproblemen der Fall ist ${ }^{3}$.

Dieser Problematik eingedenk, formulierte Broch seine «Poetik» des «polyhistorischen Romans», die den Zeitbezug jenseits eines platten Realismus oder Naturalismus und jenseits einseitiger ideologischer Prämissen der von ihm attackierten Tendenzliteratur herzustellen sucht. Der folgende Beitrag beabsichtigt nicht, die Poetik des «polyhistorischen» Romans nachzuzeichnen - dies ist an anderer Stelle bereits ausführlich geschehen ${ }^{4}$-, sondern mit Hilfe von Hermann Brochs poetologischen und 
philosophischen Essays die Umsetzung des Zeitgeschehens in Kunst und Literatur zu problematisieren und (ideologie-)kritisch zu hinter fragen.

\section{Von der «schönen» Endlichkeit des Kitsches}

3 Sie werden sich sicher schon immer gefragt haben, was Courths-Maler mit Josefine Mutzenbacher und die beiden mit Sport und Sozialismus gemein haben. Hermann Broch hält darauf eine Antwort parat:

[...] wenn also heute die Kunst und besonders die Dichtung mehr denn je zum Tummelplatz für alle möglichen fremden Wertsysteme geworden ist, wenn es nicht nur patriotrische und sozialistische Tendenzkunst gibt, sondern ebensowohl sportliche und sonstige Fachromane, so ist dies auf einen gemeinsamen Nenner zu bringen, der vielleicht am deutlichsten dort sichtbar wird, wo die Liebesdichtung in Pornographie umschlägt, das heißt dort, wo das erotische Wertsystem dogmatisch wird und die Dichtung in erotische Tendenzkunst verwandelt: das unendliche Ziel der Liebe wird ins Endliche gerückt, wird zu einer Reihe rationaler Geschlechtsakte. Und nicht anders ist es, wenn auch weniger kraß, wenn Zola das lebendige Wertsystem des Sozialismus - und damals war es noch jung und lebendig - utopisch auf das Jahr und die Verhältnisse von 1890 zusammendrängt ${ }^{5}$.

Hermann Brochs Bestimmung der Tendenzkunst geht offensichtlich weit über die gängige Besetzung des Begriffs hinaus. Mit «Tendenz» ist nicht mehr allein der Bereich der politischen Ideologien gemeint, wie etwa in der wohl griffigsten und meist zitierten Definition «schlechter Tendenzliteratur» aus der Feder Brechts:

Man bekommt «tendenziöse» Darstellungen, d.h. Darstellungen, welche allerhand auslassen, die Realität vergewaltigen, Illusionen erzeugen sollen. Man bekommt mechanische Parolen, Phrasen, unpraktikable Auweisungen ${ }^{6}$.

Ist bei Brecht der Begriff «Tendenz» auf den Bereich politischer Ideologien beschränkt, so weist bei Broch Tendenz auf eine bestimmte Lebenshaltung.

6 An dieser Stelle ist eine kurze Erläuterung der Begriffe Wert und Wertsystem bei Broch notwendig7. Er definiert ein Wertsystem als Forderungen und Arbeitsweisen, «die allesamt darauf hinzielen, den bestehenden schlechten Zustand durch einen besseren zu ersetzen $»^{8}$. Die Werte sind, einmal definiert, auf ein Partialsystem bezogen. Die Bestimmung des Wertes kann immer erst post festum erfolgen und einem «sachgebundenen Weltbild» untergeordnet werden. Einem jeden sachgebundenen Weltbild entspricht ein solches Wertsystem. Jedes Wertsystem, wenn es seine intakte Dynamik bewahrt, bezeichnet Broch als ethisch: es zeitigt auf Veränderung gerichtete Handlungen, ist lebensbejahend. Ethisch ist also nicht gleichbedeutend mit Moral und dem ihr inhärenten «du sollst». Jedes Wertsystem tendiert jedoch $\mathrm{zu}$ der Konkretisierung der ihm entspringenden ethischen Handlungen, dazu, ihnen einen festen Ausdruck zu geben. In dieser Konkretisierung wird es ästhetisch, d.h. es erstarrt in der Form, es wird verendlicht ${ }^{9}$. Broch spricht von Einzelsystemen, deren Ausdifferenzierung in der Neuzeit mit der «Auflösung des im Religiösen ruhenden ästhetischen Wertpols» (Weltbild, p. 96) erfolgt sei. Die Haltung der Verendlichung des Irdischen sei in der Romantik verwurzelt, die nach einem Ersatz für den verlorenen Wertpol, über den noch das Mittelalter verfügte, strebe ${ }^{10}$. So bezeichnet Broch in seinem Roman Pasenow oder die Romantik das an starre - ästhetische-Konventionen gebundene Leben der Offizierskaste einer zum Untergang verurteilten Epoche als romantisch (Pasenow, p. 23). 
7 An anderer Stelle definiert Broch den Kitsch als eine Tendenz zur «Systemverendlichung», die ihre Wurzel in dem romantischen Bestreben zur «Erhebung des Irdischen ins Ewige» ${ }^{11}$ habe; Kitsch ist also gleichbedeutend mit der Transposition des Ethischen ins Ästhetische; Tendenz und Kitsch gründen in derselben Lebenshaltung. Der Kitsch artikuliert ebenso die Sehnsucht nach einer klaren Orientierung in einer unübersichtlich gewordenen Wirklichkeit (Das Böse, p. 151) wie etwa die reduktioni-stischen Formeln totalitärer Ideologien. Bertolt Brecht hat in seiner Definition der Tendenzkunst das Moment der Wiederholung hervorgehoben. Das Moment der Wiederholbarkeit eignet auch dem Ästhetischen bei Broch. Kitsch und politische Phrasen sind jeweils Imitationssysteme (Das Böse, p. 153), welche rationale «Triebbefriedigung» im wörtlichen Sinne wie auch die Erfüllung des Wunsches nach Sicherheit ermöglichen (Weltbild, p. 100). Wirkungsästhetisch markiert der Effekt in die Kunst vordringender Fremdsysteme Tendenz und Kitsch:

[...] jedes dieser Fremdsysteme verlangt nach einem «Effekt», das gestrige (akademische) nach einem «schönen», das das soziale nach einem «revolutionären» oder sonst nach einem «ethisch hochwertigen», während für das Ethos der echten Kunst (mit Ausnahme der schauspielerischen) die Sünde gerade beim Effekt beginnt $[\ldots]^{12}$.

8 Der Kitsch bezieht sich nicht allein auf bestimmte Phänomene des künstlerischen Ausdrucks, die am jeweiligen Objekt nachweisbar sind; Kitsch meint bei Broch in erster Linie eine Disposition, die das rezipierte Objekt vergewaltigt. Er spricht daher vom «Kitsch-Menschen»(Bemerkungen, p. 158) ${ }^{13}$. Die Disposition ist gekennzeichnet durch die Erwartung einer raschen - inauthentischen - Befriedigung latenter oder manifester Bedürfnisse.

9 Der Kitsch-Begriff Brochs erlaubt folglich das Durchdringen künstlerischer Ausdrucksformen ebenso wie die ideologischer Konstrukte. Die politische Seite des «Kitsch-Menschen» par excellence - wir extrapolieren an dieser Stelle - ist die völlige Übereinstimmung mit einer totalitären politischen Ideologie und ihren reduktionistischen Lösungsangeboten. Brochs Werttheorie - ungeachtet ihrer letztlichen Rückbindung im Irrationalen gründenden Aporie - erweist sich, wie im folgenden am Beispiel des Gesamtkunstwerks zu zeigen sein wird, angesichts der nationalsozialistischen Kunstideologie und -politik als Instrument einer konservativen Ideologiekritik.

\section{Vom Gesamtkunstwerk und von der «schönen» Nackten ${ }^{14}$}

Das 19. Jahrhundert, insbesondere aber die Periode der Gründerzeit, zeichne sich durch ein «Wert-Vakuum» aus. In der Wissenschaft seien Fortschritte gemacht worden, die Kunst aber - mit Ausnahme der Musik - habe es nicht vermocht, die Gesamtschau dieser Epoche in den einzelnen Gattungen zu leisten ${ }^{15}$. Wagners «Gesamtkunstwerk» falle in eine «Epochen-Totalität», die um dieses «Wert-Vakuum» zentriert sei:

Er [Wagner] wußte, daß mit jedem Material sich Kunstwerke erzeugen lassen, sofern hierzu eine materialgemäße Architektonik verwendet wird, und als das Theatergenie, das er war, wußte er, daß in dem Theater und in der Oper seiner Zeit bereits Elemente vorbereitet lagen, die durch Übersteigerung ins Unromantische und dabei Überrationale, ja wenn's sein mußte sogar ins Untheatralische und Antitheatralische (wie z.B. im Tristan), zur Konstruktion der benötigten Vakuum- 
Architektur verwendbar zu machen waren; es ging um die Konstruktion des

«Gesamtkunstwerkes». (Hofmannsthal, p. 140) es zum Ausdruck des Wertvakuums seiner Epoche wird und die explizite Konkretisierung aktueller Bezüge ausblendet. Die Transposition dieses Wertvakuums erfolgt nun in dem Arrangement künstlerischer Materialien, das sich der Setzung von Werten verweigert. Darin erkennt Broch das Genie Wagners, der auf das Bedürfnis seiner Zeitgenossen nach einem kultischen Zentrum reagiert habe. Allein das Konstrukt des Wertvakuums kann - nach Broch- das Wagnersche Musikdrama vor dem Kitschvorwurf retten, doch die Aporie des Wagnerschen (Euvres in Theorie und Praxis vermag es nicht aufzuheben. Gerade das Gesamtkunstwerk, das seine Wirkung letztlich aus dem Arrangement von Effekten gewinnt, bietet sich dazu an, mit verendlichten Wertsystemen aufgeladen zu werden und auf diese Weise rasche «Befriedigung» $\mathrm{zu}$ verheißen. Der Gedanke des Wertvakuums bedeutet die Unmöglichkeit einer Befriedigung garantierenden Verendlichung von Wertsystemen am isolierten Gestaltungsobjekt und die damit verbundene Befriedigung des «Kitsch-Menschen». Der bei Broch unpräzis gebliebene Begriff des Wertvakuums umschreibt offensichtlich zum einen eine Situation, in der die Beliebigkeit und Austauschbarkeit der ästhetischen Erscheinungsformen von Werten in ein System der Unübersichtlichkeit zu münden droht, in dem das verendlichte Einzelwertsystem die rasche (scheinhafte) Befriedigung am Einzelobjekt verweigert, zum anderen eine Situation, in der die Gesamtheit dieser ästhetischen Erscheinungsformen ihrerseits als endliches System gefaßt werden. Befriedigungsmöglichkeit verheißt also das Zusammenwirken dieser «werttragenden» Objekte im Arrangement. Folgt man nun der Brochschen Argumentation, daß das Wagnersche Musikdrama eine Leerstelle ausfülle, kann, mit Hilfe der von uns vorgenommenen Erweiterung dieser Argumentation, seine Vereinnahmung durch die Nazis mit einem Quidproquo von Ethos und Ästhetik erklärt werden. So heißt es bei Broch:

[...] für ihn [Wagner] war jedes etwa noch mögliche Vakuum durch das Gesamtkunstwerk ein für allemal ausgefüllt, und die Götterdämmerung, im Grunde eine Verherrlichung des deutschen Seins und des deutschen Schicksals, war eine Theaterapotheose, deren Übertragung aufs Leben höchstens ein unerfüllbarer Wunsch sein konte. Doch jene, die nachfolgten, kurzum die Philisterbestien, vermochten das ethische Nein des Propheten nicht mehr vom Ja des TheaterÄstheten unterscheiden, und so haben Nietzsche und Wagner in seltsamer Gemeinsamkeit eine für den einen wohl mißfällige, für den anderen vermutlich wohlgefällige geistige Verantwortung für die schmählichen Ereignisse aufgelastet erhalten, welche nach nicht zu langer Frist Deutschland und die ganze Weltzivilisation erschüttern sollten. (Hofmannst hal, pp. 144 sq.)

$\mathrm{Da}$ bei Broch ein gewisses Unbehagen an dem Konstrukt Gesamtkunstwerk zu verzeichnen ist, liegt in der Gefahr des Umschlagens von einem ethischen Nein in ein ästhetisches Ja. Das Quidproquo von Et hos und Ästhetik wird möglich, da das Gesamtkunstwerk seinen architektonischen Charakter leugnet. Indem es die in ihm enthaltene künstlerische Arbeit negiert ${ }^{16}$, negiert es jeglichen Weltbezug. An die Stelle postiver Wertzetzung und dem Erfassen der Epochen-Totalität in einem Wertsystem, das deren ethisches Fundament bildet, tritt nun das Ästhetische, das als das ÄsthetischBöse die politisch-soziale Wirklichkeit definiert. Brochs frühe Analyse des Epochenstils erscheint aus der Retrospektive geradezu eine Warnung vor dem politischen Mißbrauch ästhetischer Begrifflichkeit ${ }^{17}$. 1933 gibt Broch einem Aufsatz den Titel Über 
das Böse im Wertsystem der Kunst ${ }^{18}$. Obwohl hier nicht explizit von der Nazi-Kunst die Rede ist, bildet diese doch eindeutig den Hintergrund, vor dem wiederholt das Mißtrauen gegenüber dem Gestalteten artikuliert und das Ästhetische mit dem Bösen assoziiert wird: so nennt er in einem 1950 gehaltenen Vortrag Hitler einen «unbedingte[n] Kitsch-Anhänger» ${ }^{19}$. Die Vordenker nationalsozialistischer Blut- und Boden-Ideologie bedienten sich häufig eines Begriffsarsenals aus dem Bereich der Ästhetik, um ihr rassisches «Ideal» zu beschreiben ${ }^{20}$. In Rosenbergs 1936 erschienenen Machwerk Die Gestaltung der Idee ist über den Stilbegriff der nationalsozialistischen Bewegung folgendes zu lesen:

Und so entwickelt sich die nationalsozialistische Revolution immer mehr zu einem kämpf der Weltanschauungen, zu einem Kampf um die Rangordnung der Werte und damit letzten Endes zu einem Ringen um einen geschlossenen Lebensstil der Nation überhaupt. Denn unter dem Begriff Stil kann nur die auf einen einheitlichen Willenskern zurückgehende Ausstrahlung in Politik, Kunst und Wissenschaft begreifen, die auch auf den äußeren Gebieten des Lebens die gleiche innere Voraussetzung des Urteils und der äußeren Haltung gibt ${ }^{21}$.

Schlüsselbegriff der nationalsozialistischen «Ästhetik» war der Begriff der Gestalt. Die reine Gestalt war Ausdruck der Rassenseele; die Rasse wurde somit als «Tiefenstruktur» angesehen, die allen Manifestationen menschlichen Lebens ihre «Gestalt» aufprägte, zum beherrschenden «Stil» wurde (Frank, p. 114). Das politisch-soziale Leben sollte ebenso von einem «Stil» durchdrungen werden wie Kunst und Wissenschaft - oder in der Begrifflichkeit Hermann Brochs: die nationalsozialistische Bewegung verkürzte alles auf ein endliches Wertsytem, sie realisierte das Ästhetisch-Böse in Reinkultur. Die nationalsozialistische «Gestalttheorie» gründete in dem Entleeren und Umdeuten des klassischen Schönheitsideals im 19. und 20. Jahrhundert, wie dies von Hermann Glaser aufgezeigt wurde:

Der Gedanke der Kalokagathie (der Harmonie von Seele und Leib, des erwünschten Zusammenklangs einer schönen Seele mit einem schönen Körper), von der Klassik aus der Aufklärung übernommen und weiterentwickelt, erfuhr schon bald im 19. Jahrhundert eine zu stark aufs Körperhafte bezogene Auslegung. Die «Schöne Lieblichkeit» war wichtiger als die Seele $[. . .]^{22}$.

14 An der Darstellung des nackten Körpers wird ersichtlich, wie das Umschlagen vom Ethischen, d.h. von der Klassik und ihrem von Totalitätserfahrung beseelten Geistes, ins Ästhetische überging und von da in die ihrer Anfüllung durch Werte harrende Leere mündete, wodurch der isolierte Akt weder in eine seine Darstellung transzendierende, ethische Erfahrung mündete noch die ästhetische Befriedigung des Kitsch-Menschen erlaubte.

Das von Hermann Glaser angeführte Beispiel verdeutlicht, was Broch die Verendlichung - man mag sagen Entseelung - eines Wertsystems nannte. In einer Epoche des Wertvakuums bleibt in den einzelnen Kunstgattungen - hier versuchen wir Broch weiterzudenken - nur noch die leere Gestalt, weshalb jene zu ihrer Aufhebung im Gesamtkunstwerk zu tendieren scheinen. Die Perversion dieses Gedankens etwa in dem rassisch-ästhetischen Anspruch nationalsozialistischer Aktdarstellungen wird deutlich, wenn Rosenberg fordert, «dem einen künstlerischen Stil geben, was noch ungeformt in Millionen Menschenherzen lebendig ist» (Rosenberg, p. 331).

Brochs Diktum vom Bösen im Wertsystem der Kunst wendet sich gegen ein Vereinnahmen ästhetischer Kategorien durch die Politik. Seine werttheoretische Annäherung an die Kunst löst vor der Folie des nationalsozialistischen Siegeszuges 
teilweise den Anspruch ein, den Walter Benjamin in seinem Aufsatz Das Kunstwerk im Zeitalter seiner technischen Reproduzierbarkeit erhob, nämlich die Kunsttheorie vor einem Korrumpieren durch den Nationalsozialismus zu bewahren und für ein Ästhetisieren der Politik unbrauchbar zu machen. Allerdings vollzieht der Konservative Broch nicht den Schritt Benjamins, der seine neuen Begriffe zur «Formulierung revolutionärer Forderungen inder Kinst-politik» verwenden will ${ }^{23}$; Brochs Kunst- und Literaturtheorie bewahrt gerade in dem Dualismus von ethisch und ästhetisch den Primat der Ästhetik (Hofmanntshal, p. 258) zur Verteidigung der Autonomie von Kunst und Literatur.

\section{Die Historisierung im Zeitroman}

Die Kategorie des Gesamtkunstwerkes bleibt an das historische Moment einer Epoche des Wertvakuums gebunden. Daher formuliert Broch den Primat der jeweiligen Einzelgattungen, die das Wert system ihrer Epoche adäquat wiedergeben, auch wenn etwa eine literarische Gattung qua Abstraktion in eine andere Kunstgattung überzugehen scheint, wodurch letztlich jeder Wahrheitsanspruch suspendiert wird.

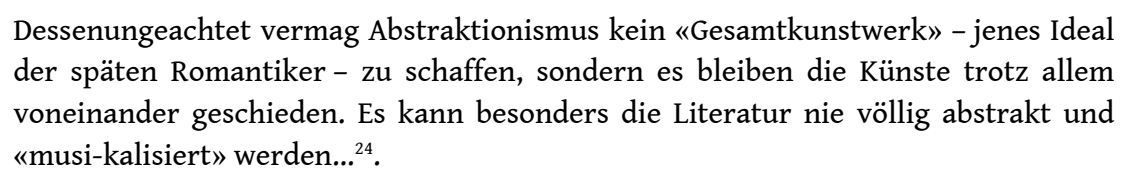

Brochs Unterscheidung in gute und schlechte Tendenzkunst zeigt, daß in einem Roman durchaus zwei Wahrheitsmomente miteinander zu konkurrieren vermögen: das verendlichte der Tendenz und ein über dieses hinauweisendes, diskursiv nicht erfaßbares. Die im Werk eingesetzten «Kunstmittel» erschöpfen sich nicht in der Wiedergabe des Tendenzmoments. Jedem Wertsystem eigne zwar das Bestreben, dogmatisch in die Dichtung einzudringen, «das Wertziel als konkret und erreichbar anzunehmen» (Weltbild, p. 98), dennoch muß dieses nicht unbedingt den Roman strukturieren ${ }^{25}$.

Broch unterscheidet zwischen dem wissenschaftlichen und dem dichterischen Wertsystem. Das wissenschaftliche Wertsystem zeichne sich durch das Streben nach einem «Maximum an Ob-jektbezogenheit» aus. $\mathrm{Zu}$ diesem Ideal strebe die Reportage. Gehen nun Elemente der Reportage in den Roman ein, so strebe dieser offensichtlich dasselbe Ideal an, denn «an Bemühungen, den zeitgenössischen Roman als historischen aufzuzäumen, hat es nicht gefehlt» (Weltbild, p. 101) Hermann Broch thematisiert hier ein Grundproblem des historischen bzw. «anderen» historischen Romans, wie es von Hans Vilmar Geppert aufgezeigt wurde. Im historischen Roman ist ein Hiatus $\mathrm{zu}$ verzeichnen, den es erst durch den Leser zu konkretisieren gilt und in dem - mit Broch argumentiert - die Differenz der beiden Wertsysteme hervortritt. Die Wahrnehmung dieses Hiatus setzt «zum einen ein voll entwickeltes Fiktionsbewußtsein, zum anderen ein Bewußtsein vom Eigenrecht und Eigengewicht der nun auch eigens $\mathrm{zu}$ definierenden historischen Fakten ${ }^{26}$ voraus. Es entsteht damit eine Dichotomie von literarischer Fiktion und dem objektbezogenen epistemologischen Standort des Historikers. Der Zeitroman der Weimarer Republik z.B., vor dessen Folie die Argumentation Brochs zu sehen ist, zeichnet sich durch das explizite Hereinziehen der zeitgenössischen Empirie in die literarische Fiktion bzw. deren Historisieren aus ${ }^{27}$. Gelangt der (historische) Objektbereich in den Roman, spricht das «Objekt selbst» so eignet diesem ein Duplizität, die in zwei konkurrierenden Wertsystemen gründet. Als 
Objekt der Geschichtsforschung oder - wir ergänzen - der Soziologie beansprucht es einen anderen Stellenwert denn als Element der literarischen Fiktion. Nichtsdestoweniger dringt mit dem objekt der Reportage auch ein Teil des diese tragenden «wissenschaftlichen» Wertsystems in den Roman ein, ohne das der Hiatus von Historie und Fiktion nicht wahrnehmbar wäre. Das Einlösen des Wertsystems der soziologischen oder historischen Wissenschaft im Roman wäre gleichbedeutend mit Verendlichung, da es ein Abgelten oder Befriedigen des jeweils an das objekt herangetragenen ethischen Anliegens suggerieren würde; der Roman würde zur Tendenzliteratur, wie in den Romanen Zolas, der «das lebendige Wertsystem des Sozialismus [...] utopisch auf das Jahr 1890 zusammendrängt». Der Zeitroman darf dann - wir extrapolieren hier wieder Brochs Argumentation - Literarizität beanspruchen, wenn er sich nicht ausschließlich auf die Verendlichung eines solchen außerliterarischen Wertsystems beschränkt. Dies bedeutet nicht, daß er nicht partiell eine solche Verendlichung vornehmen darf. Auf diese Weise gelangt Hermann Broch zur Unterscheidung von schlechter und guter Tendenzliteratur. Hierin liegt der Unterschied zu Brecht begründet, der am Beispiel der Internationalen das unablässige Sich-Bewähren in revolutionären Situationen als Kennzeichen gelungener politischer Dichtung hervorhebt.

20 Wie faßt Hermann Broch die Unsetzung des mit einem dichtungsfremden Wertsystem verknüpften Objekts im Roman? Er gebraucht dafür den Begriff des «Realitätsvokabulars»: «ein Vokabular, das für bestimmte Situationen bestimmte allgemeingültige Ausdrucke verwendet» (Weltbild, p. 105). Broch vergleicht nun das Aufgehen der Realitätsvokabel mit dem Eindringen der Realität in den Traum:

Aber es sind Realitätsvokabeln, die das Material der Dichtung ausmachen, und gleich dem Traum gewinnt Dichtung in neuer und eigener, und jetzt dürfen wir auch sagen, in subjektiver Logik und Syntax aus der Zusammenstellung dieser Vokabeln den Sinn, die Wirklichkeitstreue, den Symbolwert ihres autonomen Bereiches. Mit anderen Worten: auf die Realitätsvokabeln hat der Dichter, hat der Träumende keinen oder bloß einen sehr geringen Einfluß, sie gehören der objektiven Sphäre an, sie sind das Stück Reportage, das in jedem Traum, in jeder Dichtung steckt, die subjektive Sphäre dagegen, in der der Träumende frei schalten darf und immer frei schaltet, das ist die Syntax, in der er die Realitätsvokabel einbaut. (Weltbild, p. 105)

21 Eingedenk der unterschiedlichen Fundierung der beiden Begriffssysteme sei hier Brochs Vergleich mit dem Traum um einige Termini aus Freuds Traumdeutung erweitert: Die Realitätsvokabeln entsprächen dann den «Tagesresten». Die dichterische Syntax wäre dann mit den bei Freud als «Traumarbeit» bezeichneten Operationen vergleichbar. Ein Weiterführen des Vergleichs ließe sich zu einer Parallele zwischen den sekundären Beabeitungen des Traumes und der Tendenzliteratur ausbauen, also zu jenem Vergewaltigen der Realität durch die Unterordnung unter ein dogmatisiertes Wert system, wodurch ein kohärentes Szeenarium entsteht:

Eine intellektuelle Funktion in uns fordert Vereinheitlichung, Zusammenhang und Verständlichkeit von jedem Material der Wahrnehmung und des Denkens, dessen sie sich bemächtigt, und scheut sich nicht, einen unrichtigen Zusammenhang herzustellen, wenn sie infolge besonderer Umstände den richtigen nicht erfassen $\mathrm{kann}^{28}$. 


\section{Hermann Brochs polyhistorische Antwort: Die Rettung durch den Dilettantismus}

22 Angesichts der zunehmenden Mathematisierung der Philosophie wird die Literatur bei Broch zum Ort des Irrationalen (Vollhardt, p. 243) das die einzelnen Realitätsvokabeln organisiert. Um die Realitätsvokabel gruppiert sich implizit das jeweils an sie herangetragene Wertsystem. Realitätsvokabeln sind nicht nur Syntagmen, welche die Realität mimetisch reproduzieren, sondern auch Wertsysteme, die für sich genommen direkt oder indirekt, d.h. qua Extrapolation, im Roman ausgemacht werden können, die aber den Roman nicht strukturieren. Als die Realitätsvokabeln des modernen Roman bezeichnet Broch die großen Wertsysteme. Er greift dabei auf den Goetheschen Roman zurück, der die Wertsysteme seiner Epoche in sich aufnehme. Der Roman sei mit Geothe polyhistorisch geworden; einer «polyhistorischen» Haltung eignet die Aporie des Dilettantismus bei Goethe:

Das unendliche, niemals erreichte Ziel der Wissenschaft, ein Totalitätsbild der Erkenntnis zu gewinnen, der unendliche, in der Realität niemals erfüllte Wunsch der Einzelwertsystem, zur Absolutheit zu gelangen und eine Vereinigung zwischen allen rationalen und irrationalen Elementen des Lebens zu erzielen, das findet in der Kosmogonie und der einheitsstiftenden Syntax des Dichterischen zwar keine reale, wohl aber symbolhafte Erfüllung. (Weltbild, p. 116)

Für den literaturwissenschaftlich geschulten Leser ergibt sich daraus das Problem, daß der polyhistorische Roman gegen jede «Von außen» an das Werk herangetragene Heuristik abgeschottet wird. Jede Heuristik, die nicht wie die Syntax des Romans über die partielle Fundierung im Irrationalen verfügt, kann nur jeweils ihr Wert system am Roman, nicht aber diesen als Ganzes konkretisieren. Der polyhistorische Roman bildet somit die symbolische Erfüllung der authentischen kognitiven Disposition eines sich dem Irrationalen nicht verweigernden Dilettantismus; anders als der «ins Geniehafte getriebene Dilettantismus ${ }^{29}$ Wagners verknüpft der "polyhistorische» Dilettantismus Korrelate rationaler Erkenntnis mit Elementen des Irrationalen zu einem Weltentwurf, der im Verzicht auf die Annahme von Abgeschlossenheit dem Ethischen sein Recht gewährt. Das Irrationale in Wagners (Euvre besteht - wie implizit aus Brochs Äußerungen hervorgeht - allein in her Illusion des Rezipienten eines im Arrangement von Effekten gelungenen Artefakts, hinter dem jeder Wirklichkeitsbegriff zurücktritt. Ein positiv gefaßter Dilettantismus als Konstituens Brochscher Romantheorie wird in der von Milan Kundera vorgenommenen Zusammenfassung des Terminus «polyhistorisch» deutlich: "Alle intellektuellen Mittel, alle poetischen Formen aufbieten, um zu beleuchten, was nur der Roman entdecken kann: das Sein des Menschen ${ }^{30}$.

Nach Hans Blumenberg hat sich der neuzeitliche Wirklichkeitsbegriff und mit ihm ein neugebildeter Begriff des Künstlers, der sein Werk in Analogie zum Schöpfergott setzt, seit dem Ausgang des Mittelalters entwickelt. Die Wirklichkeit in der Neuzeit trete nunmehr in der Stimmigkeit einer Syntax von Elementen (der Schöpfung) in Erscheinung, sie sei ein Kontext:

Wenn es nur so etwas wie eine Eigenwirklichkeit ästhetischer Gegenstände geben kann, so stehen auch diese nicht nur unter dem Kriterium des Kontextes als Wirklichkeitsausweis, sondern auch unter der bestimmenden Notwendigkeit, hinsichtlich der Umfanges, der Weite und des Reichtums der einbezogenen Elemente mit dem Kontext Natur zu konkurrieren, also zweite Welten zu werden - 
und das heißt: nicht mehr Wirklichkeiten aus der einen und einzigen Wirklichkeit nachahmend herauszuheben, sondern nur noch Wirklichkeitswert der einen vorgegebenen Wirklichkeit als solche nachzubilden ${ }^{31}$.

Diese Scheidung der beiden Welten tritt an der Einzelgattung hervor, wobei der Roman als die «welthafteste Gattung» am weitesten vordringt, jedoch unter der Voraussetzung seines Erhalts als Gattung, denn

Die potentielle Unendlichkeit des Romans ist zugleich seine aus der Wirklichkeit bezogene Idealität und das ästhetische Ärgernis, das er unaufhebbar gibt, indem seine nur amorph zu lösende Aufgabe wiederum unter dem ästhetisch unabdingbaren Prinzip der Form steht». (Blumenberg, pp. 21 sq.)

Blumenbergs Argumentation gipfelt in der Auffassung, daß der Roman untrennbar mit dem Wirklichkeitsbegriff der «immanenten Kohärenz», Grundlage der Kontextbildung, verknüpft sei. Die logische Konsequenz muß daher lauten: Der Roman ist als Gattung nicht hintergehbar. Der Wirklichkeitsbegriff der immanenten Kohärenz disponiert auch die Rezeptionshaltung des Brochschen Kitsch-Menschen: in der raschen Suggestion von «immanenter Kohärenz» ruht die Quelle seines Befriedigungserlebnisses.

In Brochs Postulat, der Roman sei «Totalitätsbild der Erkenntnis», findet ein Antizipieren der Ecoschen Rede vom Joyceschen Roman (insbesondere Finnegans Wake) als «epistemologische Metapher» ihre Vor wegnähme ${ }^{32}$. Jedoch kann das Konstrukt des «polyhistorischen Romans» nicht als gattungspoetisches bezeichnet werden, würde es doch in letzter Konsequenz die Gattung Roman als solchen aufheben. Mit dem vollständigen poetologischen Erfassen würde der polyhistorische Roman seinerseits ästhetisch umgedeutet. Folgerichtig läßt sich dieser nur als eine Leerstelle definieren ${ }^{33}$.

Brochs These vom «polyhistorischen Roman» stellt, in ihrem historischen Kontext verortet, ein konservatives Konstrukt dar, das - literatursoziologisch gesprochen - auf die im Roman konkretisierten Vermittlungsschichten verweist ${ }^{34}$. Diese These gründet aber auf einem im Irrationalen verankerten Organisationsprinzip. Die Rede vom «polyhistorischen Roman» setzt den Erhalt der Gattung Roman und der für diese konstitutiven Autonomie von Kunst und Literatur voraus; sie erweist sich als konservativ im Hinblick auf die historische Avantgarde im engeren Sinne, die sich als Agens des Wertzerfalls manifestiert.

\section{NOTES}

1. - Schriften von Hermann Broch werden im folgenden nach der von Michael Lützeler bei Suhrkamp (Frankfurt/Main) besorgten Kommentierten Werkausgabe zitiert (KW \& Erscheinungsdatum).

2. - Broch, Die Schlafwandler. Eine Romantrilogie: KW11978: (Der erste Roman), 1888. Pasenow oder die Romantik, (Der zweite Roman) 1903. Esch oder die Anarchie (Der dritte Roman), 1918. Hugenau oder die Sachlichkeit. Cf. Ziolkowski, Theodore, Zur Entstehung und Struktur von Hermann Brochs «Schlafwandlern ", in Brude-Firnau, Gisela (Hrsg.), Materialien zu Hermann Brochs «Die Schlafwandler», Frankfurt/Main: Suhrkamp (ed.), 1972, p. 126-162. 
3. - Lukåcs, Georg, Die Theorie des Romans. Ein geschichtsphilosophischer Versuch über die Formen der großen Epik, Darmstadt/Neuwied: Luchterhand (Sammlung L.), 9. Aufl. 1984, p. 61.

4. - Cf. z.B. Steinecke, Hartmut, Hermann Broch und derpolyhistorische Roman. Studien zur Theorie und Technik eines Romantyps der Moderne, Bonn: Bouvier 1968; Durzak, Manfred, Hermann Broch. Der Dichter und seine Zeit, Stuttgart: Kohlhammer 1968, p. 76-113; Vollhardt, Friedrich, Hermann Brochs Literaturtheorie, in Lützeler, Paul Michael (Hrsg.), Hermann Broch, Frankfurt/Main: Suhrkamp 1986, p. 272-288.

5. - Broch, Das Böse im Wertsystem der Kunst, in KW IX/2 1975, p. 119-157, hier: p. 149.

6. - Brecht, Bertold, Die Dialektik, in Gesammelte Werke XIX, Frankfurt/Main: Suhrkamp (ed) 1967, p. 394 sq., hier: p. 394.

7. - Cf. die unter dem Titel Wert- und Geschichtstheorie zusammengefaßten Abhandlungen in Broch, KWX/2 1977, p. 11-203. Zu einer fundierten Darstellung der Philosophie Brochs, v.a. auch im Hinblick auf ihre Beziehung zum Neukantianismus Rickerts cf. Vollhardt, Friedrich, Hermann Brochs geschichtliche Stellung. Studien zum philosophischen Frühwerk und zur Romantrilogie «Die Schlafwandler» (1914-1932), Tübingen: Niemeyer (Studien zur deutschen Literatur) 1986.

8. - Broch, Das Weltbild des Romans, in KW IX, 2, p. 89-118, hier: p. 89.

9. - Die vom schulphilosophischen Gebrauch abweichende Verwendung der Begriffe ethisch und ästhetisch findet sich bei Søren Kierkegaard (cf. das Kapitel «Das Gleichgewicht zwischen dem Ästhetischen und dem Etischen in der Herausbildung der Persönlichkeit», in Entweder/Oder, 2. Teil, Düsseldorf: E. Diedrichs 1956, p. 165-364) vergegeben. Im folgenden sind die beiden Termini kursiv gesetzt, wenn sie im Sinne von Broch verwendet werden.

10. - In der Verbindung von Wert- und Geschichtsphilosophie stützt sich Brochs Ansatz offensichtlich auf die 3-Stadien-Lehre von Claude Henri de Saint-Simon und Auguste Comte.

11. - Broch, Einige Bemerkungen zum Problem des Kitsches, in: KW 1X52, p. 158-175, hier: p. 169.

12. - Broch, Hofmannsthal und seine Zeit, in: KWIX/1 1975, p. 111-184, hier: p. 258.

13. - U.a. auf Hermann Broch stützt sich die Studie von Ludwig Giesz, Phänomenologie des Kitsches, München: Fink (Theorie und Geschichte der Literatur und der schönen Künste 17) 2. Aufl. 1971.

14. - Dieser Abschnitt knüpft an folgenden Beitrag an: Kuhnle, Till R., Anmerkungen zum Begriff Gesamtkunstwerk - Die Politisierung einer ästhetischen Kategorie, in Germanica 10, 1992, p. 35-50.

15. - Cf. dazu die Ausführungen zu Drama und Roman bei Richard Wagner, Das Schauspiel und das Wesen der Dichtkunst, in: Ders., Dichtungen und Schriften VII, Frankfurt/Main: Insel 1983, insbes. pp. 124-176. Eine zusammenfassende Darstellung findet sich bei Gerhard von Graevenitz, Mythos. Die Geschichte einer Denkgewohnheit, Stuttgart: Metzler 1987, p. 274.

16. - Cf. Adorno, Theodor W., Versuch über Wagner, Frankfurt/Main: Suhrkamp (st) 2. Aufl. 1981.

17. - Cf. Broch, Wert- und Geschichtstheorie, p. 45-80.

18. - Osterle, Heinz D., Die Schlafwandler: Revolution und Apokalypse, in: Brude-Firnau, Materialien zu Hermann Brochs Schlafwandler, p. 151-162, hier: p. 161, macht bei dem Konservativen Hermann Broch erst nach 1933 die definitive Abkehr von kryp-tofaschistischen Neigungen aus. Broch selbst spricht - ohne den Faschismus direkt zu nennen - vom «System des Reaktionären im Wertsystem des Konservativismus» (Das Böse, p. 147).

19. - Broch, Einige Bemerkungen zum Problem des Kitsches, in: KW IX/2, p. 158-173, hier: p. 171.

20. - Cf. Frank, Manfred, Gott im Exil, Vorlesungen über die Neue Mythologie, Frankfurt/Main: Suhrkamp (ed) 1988, p. 105 - Frank hat darauf hingewiesen, daß die führenden Köpfe der nationalsozialistischen Bewegung häufig über eine künstlerische oder kulturwissenschaftliche Vorbildung verfügten.

21. - Rosenberg, Alfred, Weltanschauung und Kunst, in: Gestaltung der Idee. Blut und Ehre II, München: Zentralverlag der NSDAP 1936, p. 330.

22. - Glaser, Hermann, Spießer-Ideologie. Von der Zerstörung des deutschen Geistes im 19. und 20. Jahrhundert und dem Aufstieg des Nationalsozialismus, Frankfurt/Main: Fischer (TB) 1985, p. 34. 
23. - Benjamin, Walter, Das Kunstwerk im Zeitalter seiner technischen Reproduzierbarkeit [zweite Fassung], in: Ders., Gesammelte Schriften 1/2, Frankfurt/Main : Suhr-kamp 1974, p. 471-508, hier: p. 473.

24. - Broch, Mythos und Altersstil, in KW IX/2, p. 212-235, hier: p. 229.

25. - Cf. Wolfgang Iser, der von der anthropologischen Disposition des Fingierens ausgehend in der Literatur den Ort sieht, an dem der Mensch seiner exzentrischen Position gewahr wird, Position, die ihn zur Inszenierung führt. Diese Inszenierung könne aber zur Besetzung tendieren, «doch wo solches geschieht, ist Literatur auf dem Weg zur Utopie, in der sich der Scheincharakter der Inszenierung verdinglicht, dessen Margerkeit davon zeugt, daß nun der Modus selbst zum Objekt geworden ist» (Das Fiktive und das Imaginäre. Perspektiven literarischer Anthropologie, Frankfurt/Main: Suhr-kamp 1991, p. 507).

26. - Geppert, Hans Vilmar, Der andere historische Roman. Theorie und Struktur einer diskontinuierlichen Gattung, Tübingen: Niemeyer (Studien zur deutsche Literatur) 1975, p. 38.

27. - Dieses Problem hat Jean Paul Sartre in seiner Schrift La Situation de l'écrivain, in: Ders. Situations II (Qu'est-ce que la littérature), p. 202-330, mit seinem Konstrukt des romans de situation weitergedacht. Cf. die in Kürze bei Niemeyer erscheinende Dissertation des Verfassers (Augsburg 1992), Chronos und Thanatos. Zum Existentialismus des Nouveau romancier Claude Simon (Kapitel: Der «roman de situation»). Ein vertiefter Vergleich zwischen Theorie und Praxis der littérature engagée und dem Zeitroman der Weimarer Republik ist nach unserer Kenntnis noch ein Desiderat der Forschung.

28. - Freud, Sigmund, Totem und Tabu (GWIX), Frankfurt/Main: Fischer: 3. Aufl. 1961, p. 117; cf. auch Ders., Die Traumdeutung (GWII/III), Frankfurt/Main: 3. Aufl. 1961, p. 492-512.

29. - Mann, Thomas, zitiert nach Adorno, Versuch über Wagner, p. 24.

30. - Kundera, Milan, Die Kunst des Romans, Frankfurt/Main: Fischer (TB) 1989, p. 73 sq. Kundera bezeichnet Stifters Nachsommer als den «polyhistorischen» Roman schlechthin, weil hinter der enzyklopädischen Gelehrsamkeit der Mensch und die menschliehen Situationen zurückblieben (id., p. 73).

31. - Blumenberg, Hans Wirklichkeitsbegriff und Möglichkeit des Romans, in: Jauss, Hans Robert, Nachahmung und Illusion (Poetik und Hermeneutik I), München: Fink 1969, p. 9-27, hier: p. 21.

32. - «Metapher wohlgemerkt: also nicht Übersetzung epistemologischer Situationen, sondern Aufzeigen strukturell analoger Situationen. In diesem Zusammenhang kann und soll das Werk übrigens nicht mit einem bestimmten System verglichen werden, dessen orthodoxes Bild es wäre: es handelt sich vielmehr darum, in ihm Motive festzustellen, die, auf - oft diskordante wissenschaftliche Erkenntnisse zurückbezogen werden [...]» (Eco, Umberto, Das offene Kunstwerk, Frankfurt/Main: Suhrkamp 1973, p. 412. Zum «erkenntnistheoretischen Roman» bei Broch cf. Kreutzer, Leo, Erkenntnistheorie und Prophétie. Hermann Brochs Romantrilogie «Die Schlafwandler», Diss. Tübingen 1966, p. 15 sqq.

33. - Cf. die Kritik Kunderas (p. 74) an dem letzten Buch der Schlafwandler-Trilogie.

34. - Zum Begriff der Vermittlung cf. Köhler, Erich, Einige Thesen zur Literatursoziologie, in: Ders. Vermittlungen. Romanistische Beiträge zu einer historischsoziologischen Literaturwissenschaft, München: Fink, p. 8-15. 


\section{RÉSUMÉS}

Mit Tendenz meint Hermann Broch nicht allein den Bereich politischer Ideologien, sondern auch ein bestimmtes Verhältnis zu Werten. Ein Wertsystem definiert er als Forderungen und Arbeitsweisen, die darauf hinzielen, den bestehenden Zustand durch einen besseren zu ersetzen. Broch unterscheidet zwischen ethischem Handeln, das auf die Veränderung des status quo ausgerichtet ist, und ästhetischem Handeln, das sich an festgelegten Regeln mißt. In seinen Essays zeichnet Broch das Bild des Kitsch-Menschen, das in der Person Adolf Hitlers gipfelt. Dabei weicht die Terminologie Brochs vom gängigen Gebrauch der Begriffe ethisch und ästhetisch ab. Er nähert sich dabei der Position Walter Benjamins an, der dem politischen Mißbrauch traditioneller ästhetischer Kategorien durch den Faschismus entgegenarbeitet. Der Konservative Broch folgt jedoch Benjamin nicht, wenn dieser eine «Politisierung» der Ästhetik durch eine neue, revolutionäre Begrifflichkeit fordert. Broch hält an dem Gedanken von der Kunstautonomie fest und lehnt das avantgardistische Choc-Prinzip zum Zwecke der Reintegration der Kunst in die Lebenspraxis ab. Nichtsdestoweniger bildet seine Werttheorie ein Instrument der Ideologiekritik.

Um den Roman gegen die völlige ideologische Vereinnahmung zu verteidigen, entwirft er seine Theorie des polyhistorischen Romans. Broch lehnt nicht von vornherein jegliche Form der engagierten Kunst $a b$, wenn die Werke zugleich über den eng gesteckten Horizont einer Tendenzkunst hinausweisen. So ist bei ihm etwa eine Wertschätzung für das Theater Brechts zu verzeichnen. Der Gedanke des Polyhistorismus, des Hereinziehens der großen Wertsysteme der jeweiligen Epoche, nähert sich dem des Dilettantismus an. Niemand - und Broch ist sich dessen bewußt - ist in der Lage die Werte seiner Epoche in ihrer Totalität zu reproduzieren.

Selon Hermann Broch, l'attitude tendancieuse englobe non seulement un jugement politique, mais qualifie également une certaine approche des valeurs. Un système de valeurs est constitué d'exigences et de règles d'action ayant pour but le remplacement d'un statu quo par un état meilleur. Broch établit une distinction entre les actions éthiques, aptes à changer ce statu quo, et les actions esthétiques qui sont confinées dans les règles de ce dernier. Dans ses essais esthétiques, Broch retrace la phénoménologie de l'homme-kitsch qui culmine dans la personne d'Adolf Hitler : l'incarnation du mal dans l'esthétique. Les réflexions de Hermann Broch s'éloignent de l'usage traditionnel des termes éthique et esthétique pour se rapprocher finalement de celles de Walter Benjamin qui récuse l'abus des catégories esthétiques traditionnelles à des fins politiques par le fascisme. Mais le conservateur ne suit pas Benjamin dans sa tentative d'une " politisation » de l'esthétique par une nouvelle terminologie révolutionnaire. Broch reste fidèle à l'idée de l'autonomie de l'art; il récuse le concept avant-gardiste de la réintégration de l'art dans la vie par le choc. Néanmoins, la théorie des valeurs constitue un instrument de la critique des idéologies.

Pour défendre le roman contre sa soumission totale à des fins idéologiques, il développe sa théorie du roman polyhistorique. Broch ne récuse pas totalement tout art engagé - comme le théâtre de Brecht -, mais seulement à condition que les œuvres en question transcendent en même temps les limites de la littérature tendancieuse. L'idée d'un polyhistorisme, cherchant à intégrer les systèmes de valeur de son époque, se rapproche de celle du dilettantisme. Nul - et Broch en est conscient - n'est en mesure de représenter la totalité des valeurs de son époque. 
AUTEUR

TILL R. KUHNLE

Universität Augsburg 